\title{
Desarrollo e innovación de los sistemas mecatrónicos en un automóvil: una revisión
}

\author{
(Development and innovation of electronic systems \\ in an automobile: a review)
}

\author{
Marco V Noroña $M,{ }^{1}$ Manuel F Gómez $B^{1}$
}

\begin{abstract}
Resumen
Este artículo presenta un estudio de los avances tecnológicos en la parte electrónica de un automóvil, se basa en un análisis de las especificaciones técnicas, económicas, productivas y medioambientales consideradas en el diseño, desarrollo e innovación de los componentes de un vehículo, que cumplan con los requerimientos de las empresas automotrices. Explica de manera sistemática la idea y contenido de los sistemas de innovación implementados en los vehículos en los últimos años, en la parte eléctrica-electrónica, tras los diversos estudios realizados por científicos, investigadores y analistas de la innovación, en el campo de la electrónica automotriz. Además, se discuten las transformaciones ocurridas en los últimos años en la parte eléctrica-electrónica de un vehículo y se elabora un análisis comparativo de las modificaciones y/o mejoras en las características de los sistemas automotrices, para cumplir con las normativas existentes respecto del consumo de energía, rendimiento, tratamiento de residuos, confortabilidad, seguridad, entre otras. La introducción de nuevos sensores, unidades de control y actuadores, relacionados con la microelectrónica, telemática y multimedia a los vehículos proporciona cambios profundos en el diseño, producción, operación y diagnóstico de un automóvil, que se han implementado paulatinamente en los sistemas de: frenos, transmisión, dirección, seguridad, confort y motor.
\end{abstract}

\section{Palabras clave}

Avances tecnológicos; mecatrónica automotriz; autónomo; innovación; redes.

\begin{abstract}
This article presents a study of the technological advances in the electronic part of an automobile, based on an analysis of the technical, economic, productive and environmental specifications considered in the design, development and innovation of components of a vehicle that meet the requirements of the automotive companies. It systematically explains the idea and content of the innovation systems implemented in vehicles in the electrical-electronic part in recent years, after several studies carried out by scientists, researchers and innovation analysts in the field of automotive electronics. The transformations occurred in the last years in the electrical-electronic part of a vehicle are discussed and a comparative analysis of the modifications and / or improvements in the characteristics of the automotive systems is elaborated, in order to comply with the existing regulations regarding energy consumption, performance, waste treatment, comfort, safety, among others. The introduction of new sensors, control units and actuators, related to microelectronics, telematics and multimedia to vehicles, provides profound changes in the design, production, operation and diagnosis of a car, which have been gradually implemented in the systems of brakes, transmission, steering, safety, comfort and engine.
\end{abstract}

\section{Keywords}

Technological advances; automotive mechatronics; autonomous; innovation; networks.

\section{Introducción}

Con base en las necesidades y especificaciones técnicas de la aplicación automotriz a fabricar, en este caso un dispositivo electrónico, se debe analizar las condiciones de funcionamiento de la pieza, tanto de manera individual como en su conjunto; para ello se toma en cuenta aspectos técnicos 
de diseño y fabricación. Como todo elemento de uso automotriz debe pasar por rigurosos métodos de evaluación y pruebas para cumplir con las especificaciones requeridas (Parera, 2000).

El fabricante, en función de las especificaciones requeridas, diseña y fabrica un vehículo que asegure cumplir con los requisitos sociales y legales, utilizando las tecnologías disponibles, que den como resultado un producto de alta calidad y al costo más apropiado (Fernández, 2017).

En los años, cuando los automóviles comenzaban a popularizarse, las prestaciones que demandaban los usuarios no eran muchas. En los años y los automóviles tenían que ser más grandes y más veloces, con precios más económicos, interviniendo el desarrollo tecnológico de la parte electrónica y, mecánica de los sistemas del vehículo. Entre 1980 y 1990 la evolución y desarrollo se basó en los sistemas de inyección electrónica de combustible y los sistemas de seguridad. A partir de 1990 hasta el año 2000 las prestaciones estaban enfocadas en la confortabilidad y navegación. Entre los años de 2000 al 2010 el auge de la tecnología se basaba en los vehículos híbridos; vehículos conectados, aunque nacen como concepto en los y empiezan a fabricarse en los. A partir de 2010 las prestaciones están enfocadas hacia los vehículos inteligentes y vehículos autónomos. Junto con el crecimiento de la tecnología de la información y la electrónica la industria automotriz se relaciona con la aplicación de tecnologías de nuevas industrias como la del internet móvil, incluyendo la digitalización y el desarrollo de los vehículos inteligentes.

Actualmente, los automóviles vienen equipados con una gran cantidad de dispositivos electrónicos que cumplen un sinnúmero de funciones desde recibir y emitir una señal hasta la automatización mediante el intercambio permanente de datos e información, por ello, la importancia del presente estudio y revisión bibliográfica de investigaciones precedentes, las cuales nos permiten analizar los sistemas del vehículo que son intervenidos por la electrónica con el fin de ayudar y mejorar las prestaciones de servicio del automotor, ya que este tema está ligado con el desarrollo de nuevas tecnologías.

El trabajo está dividido en tres partes. En la primera, se tratan los orígenes, la evolución e importancia de los avances e innovaciones en los sistemas eléctrico-electrónicos de los vehículos y la relación existente con los avances tecnológicos de los sectores electrónico y automotriz. En la segunda parte se analiza cómo influyen estos sistemas en el comportamiento y funcionamiento de los diferentes sistemas del vehículo. En la tercera parte se profundiza el análisis de las aplicaciones de los sistemas mecatrónicos y sus ventajas, desde la perspectiva de los diversos autores consultados. Es por todo esto que el objetivo de este trabajo es explicar la evolución que está experimentando el sector del automóvil y cómo se relaciona con el desarrollo e implementación de dispositivos electrónicos (Lucas, 2016).

\section{Desarrollo}

El presente artículo analiza los avances tecnológicos y de innovación del automóvil que van orientados hacia el uso y reutilización de materiales ligeros, seguridad y confortabilidad, vehículos inteligentes y/o autónomos, movilidad sostenible, eficiencia energética y sustentabilidad, lo que ha ocasionado que la electrónica tome una gran importancia. La metodología utilizada en este trabajo tiene su soporte en fuentes bibliográficas, en donde tras el análisis de varios textos bibliográficos sobre la evolución de la electrónica de consumo que ha dado lugar a requisitos más exigentes para las comunicaciones y las funciones de entretenimiento de los vehículos. Uno de los textos que más se analiza es el de Lara Rivero del año 2014 en donde se estudia la innovación y evolución de las funciones electrónicas en un automóvil. 


\subsection{Electrónica en el automóvil}

La electrónica en la automoción desempeña un papel muy importante. Los productos electrónicos incorporados en un vehículo, en la actualidad, superan la tercera parte del valor del mismo.

Desde la década del sesenta, con los primeros encendidos con transistores, la industria electrónica ha ocupado un campo cada vez más amplio y variado en la industria automotriz. Millones de sensores, de elementos electromecánicos y micromecánicos se fabrican cada año para cubrir las necesidades crecientes de la industria automotriz. Cada vehículo incorpora múltiples dispositivos electromecánicos para actuar en las áreas de eficiencia energética, operación, asistencia a la conducción, seguridad, confort y disminución de la polución.

Durante los últimos años gran parte de las innovaciones incorporadas a los vehículos automotores están relacionadas con la electrónica. Y la tendencia es que estas proyecciones continúen ya que la tecnología electrónica provee el medio más eficiente y, en algunos casos, el único disponible para el logro de las mejoras funcionales que se planea incorporar a los vehículos en el futuro cercano (Schöner, 2004).

Otro factor importante es la visión ambiental, por las normas que a nivel nacional y/o internacional deben cumplir los automóviles, y por tanto el uso de la electrónica se puede traducir a ventajas competitivas al disminuir el consumo de materiales y energía, reutilización y reciclado porque se reducen costos.

Los requisitos y condicionantes implicados en el diseño y construcción de un vehículo a la hora de que un fabricante lo diseñe y lo ponga en producción, se ven influidos por una serie de requisitos o condiciones que ha de tener en cuenta para que el automóvil tenga posibilidades comerciales, y estos requisitos son del tipo: sociales, legales y tecnológicos.

La situación actual, las principales empresas manufactureras de automóviles considerarán a la electrónica como el factor clave para competir por un mercado de clientes. La innovación se está volviendo cada vez más interdisciplinaria, involucrando industrias como las ciencias de los materiales (materiales ligeros), la industria química (baterías) y la electrónica (comunicaciones y sistemas de entretenimiento).

El sector automotriz tiene una importante participación en la industria electrónica:

- Cerca del $9.5 \%$ de la venta de semiconductores (The Semiconductor Industry Association, 2014).

- El14\% de la producción de tarjetas de circuitos impresos. (Fiarchild Semiconductor, 2012)

- Los componentes electrónicos constituían el $1 \%$ del costo de un automóvil a finales de los setenta; actualmente representan el $40 \%$. (Industria CCOO, 2018)

- El desarrollo de nuevos productos integra procesos de convergencia tecnológica, que son analizados por estudios conjuntos entre grupos de empresas (Greenstein, 1997).

La integración de la electrónica en un vehículo se relaciona con:

1. Cambio de elementos y/o componentes. Funciones que realizaban los sistemas mecánicos fueron reemplazadas por sistemas mecatrónicos. Por ejemplo, el sistema de inyección electrónica sustituyó al carburador, entre otros.

2. Adaptación de nuevas funciones. A las funciones normales se les integra sistemas mecatrónicos que proporcionan mejor rendimiento. Principalmente en los sistemas de seguridad, confortabilidad, navegación, y comunicaciones. Por ejemplo, el sistema de bolsas de aire, los frenos antibloqueo, entre otros. 
3. Procesos de hibridación. Funciones viejas se combinan con una parte nueva. Por ejemplo, sistemas de propulsión híbridos, entre otros.

La electrónica del automóvil ha obtenido desarrollos en los siguientes sistemas del vehículo (Cook et al., 2006):

- Motopropulsión

- Seguridad

- Confortabilidad

En la Figura 1 se puede observar la evolución que han tenido los diferentes sistemas del vehículo con la aplicación de la electrónica en sus componentes.

En los años sesenta, se inició con la primera etapa, en la cual se realizaban trabajos de sustitución de partes mecánicas por partes electrónicas con el objetivo de mejorar la fiabilidad. A manera de ejemplo se tiene, en 1965, el encendido por ruptor dio paso al encendido transistorizado.

Figura 1. Innovación y evolución de las funciones electrónicas en un automóvil (Lara, 2014)

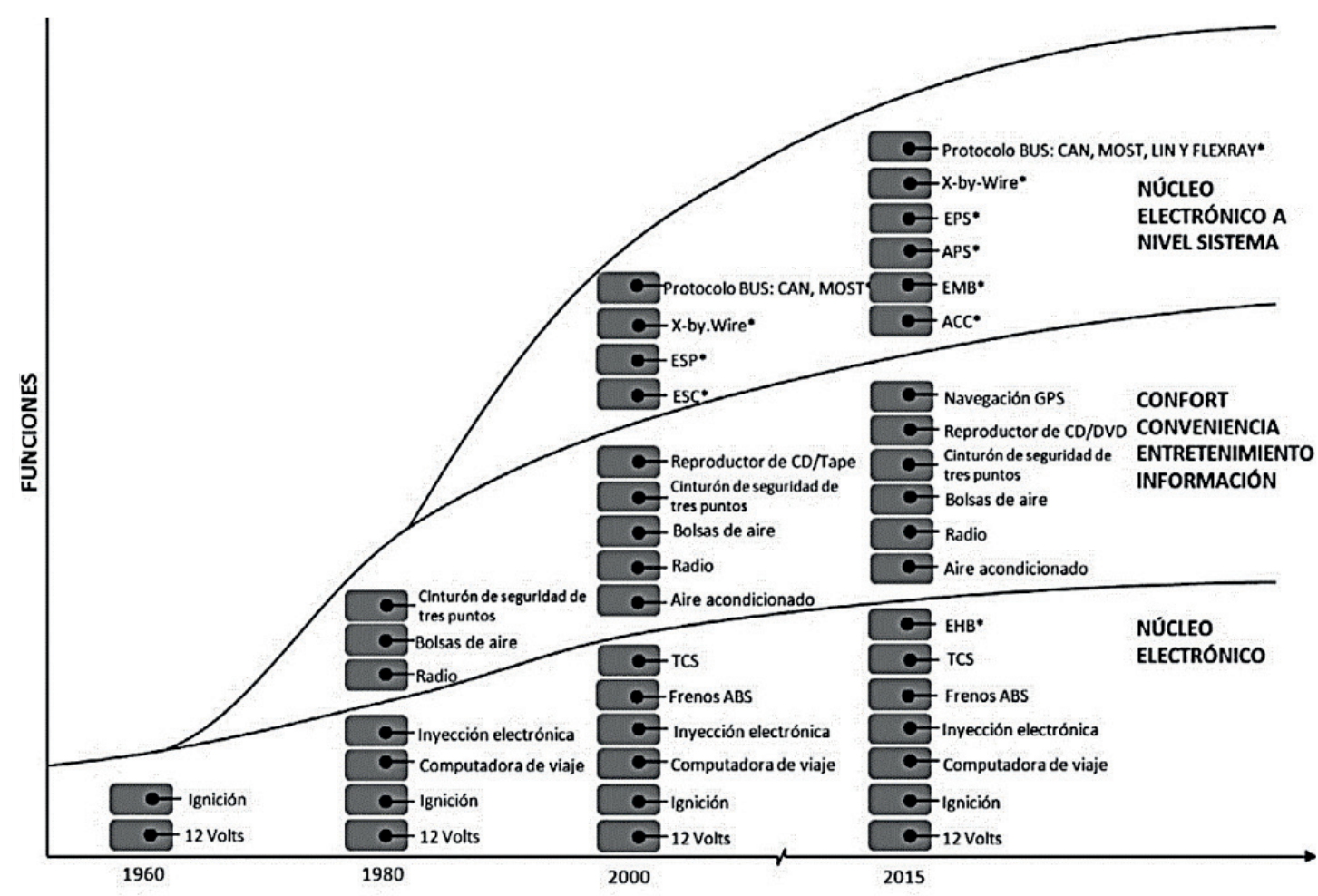

Notas: ABS - Sistema de Frenos Antibloqueo; ESP - Programa Electrónico de Estabilidad; TCS - Sistema de Control de Tracción; EHB - Frenos Electro Hidráulicos; ESC - Control Electrónico de Estabilidad; CAC - Control de Navegación Adaptable; EPS - Sistema Dirección Electrónica; EMB - Frenos Electro Mecánicos; APS - Dirección Asistida; X-by-Wire Incluye: Conducción-por-Cable, Dirección-por-Cable, Frenos-por-Cable, Transmisión-por-Cabley Suspensión-por-Cable; CAN-Controlador de Área de Red; LIN-Red de Interconexión Local; MOST - Medios de Comunicación orientada a transporte de sistemas; FLEXRAY-Protocolo de comunicación BUS. - En desarrollo.

Hasta la década de los sesenta un vehículo convencional estaba básicamente conformado por componentes mecánicos; la integración de componentes electrónicos inicia su ascenso en los años setenta. Si se compara un vehículo típico de los años sesenta con los vehículos actuales, las diferencias cuantitativas son significativas, pero las transformaciones cualitativas son más profundas. 
Durante el período 1960-1969, las empresas del sector automotriz experimentaron el uso de componentes electrónicos. En 1960, Electric Autolite y Delco-Remy iniciaron el uso de transistores con el propósito de regular la energía eléctrica de los interruptores (breaker points).

La aparición de los cinturones de seguridad y su posterior desarrollo legislativo hace que, desde la década de los setenta, comience a ser de aplicación la legislación que hace referencia al diseño e instalación de asientos y cinturones de seguridad en el vehículo. En primer lugar, surgieron los cinturones de seguridad automáticos y, posteriormente, los airbags. En los años noventa, debido al desarrollo tecnológico, los vehículos comenzaron a incorporar importantes innovaciones en materia de seguridad activa, como el ABS (Sistema antibloqueo de ruedas, del alemán Antiblockiersystem) y el ESP (control de estabilidad).

A finales de los setentas, el aire acondicionado fue incluido en la mayoría de los vehículos. Este fue el primer paso hacia el asombroso confort térmico que han alcanzado los coches del presente.

A principios de la década de los setenta tuvieron su desarrollo los microprocesadores o unidades de control electrónico (Electronic Control Unit, ECU, por sus siglas en inglés).

En Estados Unidos, la "California Air Resources Board" determinó en 1988 que todos los automóviles a gasolina contaran con un elemento que les permitiera realizar diagnósticos del motor. De ahí nacen los OBD (On Board Diagnostics), que permiten controlar los límites máximos de emisiones mediante dispositivos de mando electrónicos.

Entre los años de 1989 y 1995, se integraron en los vehículos sistemas mecatrónicos de control de la tracción y programas electrónicos de estabilidad, respectivamente. El primer componente del sistema " $X$-by-wire" introducido a la industria fue el "acelerador-por-cable" a fines de los noventa y principios del año 2000 (Bonet, 2017).

La incorporación de sistemas mecatrónicos en los vehículos permitió también llenar de electrónica los captadores y los actuadores (Figura 2). Cada vez más asentada y extendida, la electrónica del automóvil experimentó un verdadero auge en los años noventa. Fue en esta década cuando se incluyeron en los vehículos de gama baja y media.

Figura 2. Electrónica aplicada al automóvil: Captadores y Actuadores (París, 2003)

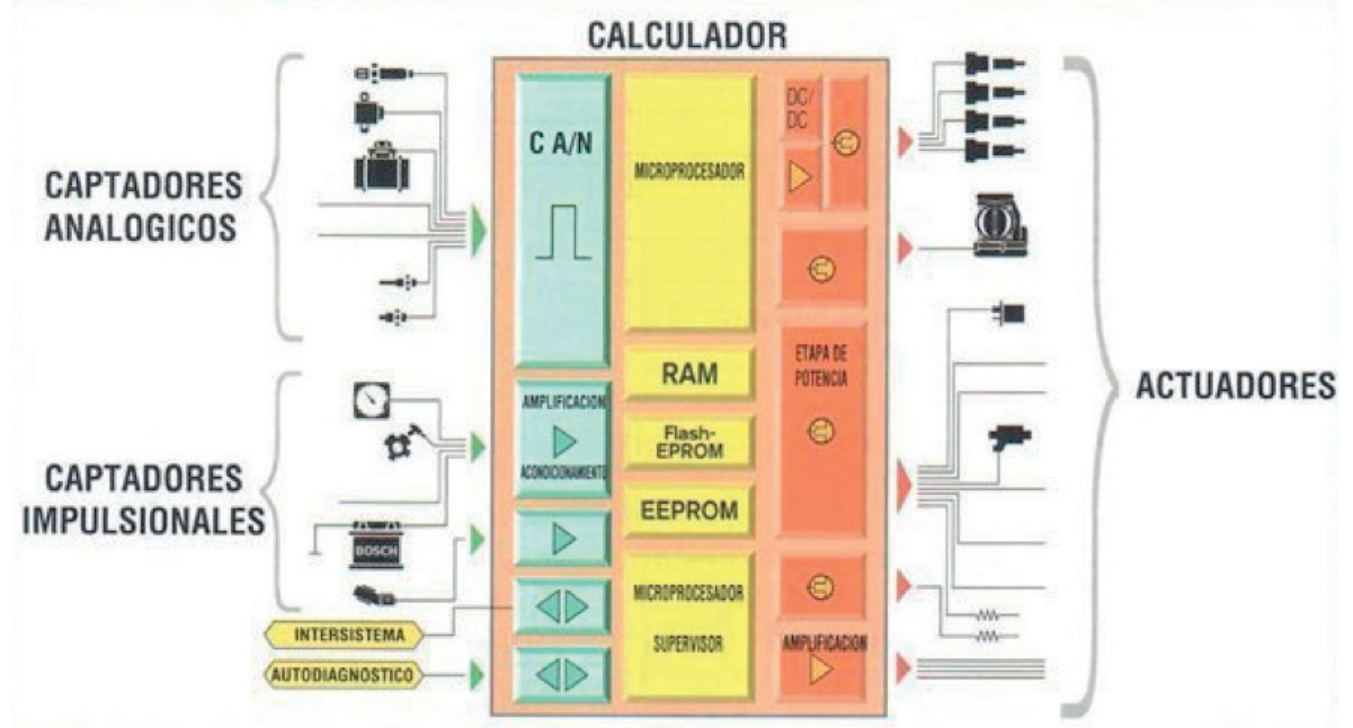


A partir del año 2000 la electrónica inició una nueva etapa al emerger la mecatrónica, cuyo objetivo es la integración perfecta de la electrónica en la arquitectura del vehículo. De este modo, la mecatrónica ha permitido la optimización de los sistemas de los vehículos, al asociar estrechamente componentes mecánicos, electrónicos y programas, tales como el ESP (Fernández, 2017).

Actualmente, los automóviles incluyen una variedad sin precedentes de innovaciones que requieren un número significativamente mayor de dispositivos electrónicos. Desde cámaras, sensores y procesadores de imagen necesarios para sistemas avanzados de asistencia al conductor (ADAS) en automóviles convencionales, hasta electrónica de potencia sofisticada utilizada en vehículos eléctricos (VE) y tecnologías avanzadas de sensores para vehículos autónomos "autónomos", automotriz y semiconductor las industrias están más estrechamente ligadas que nunca (Lammers, 2008).

\subsection{Sustitución de las partes mecánicas por componentes electrónicos}

Varias de las funciones mecánicas han sido sustituidas por componentes electrónicos, entre las más importantes existen: la sustitución de sistemas mecánicos e hidráulicos por componentes eléctrico/electrónicos (E/E) se describe con el término genérico "X-by-wire" (Leen \& Heffernan, 2002). Se ha identificado el reemplazo de más de nueve funciones vinculadas con la aceleración, el frenado, el cambio de velocidades, la suspensión, la integración encendido/alternador, el control variable de válvulas, el convertidor catalítico, los dispositivos eléctricos, así como con otros accesorios eléctricos. Todos ellos generan señales que van a diferentes microprocesadores dedicados que toman decisiones para la operación del vehículo. Entre los principales desafíos de la electrónica del automóvil está su robustez para soportar las exigencias dinámicas a las que está sometido el vehículo, así como a los requerimientos de exigentes condiciones ambientales de operación. En la Figura 3 pueden apreciarse los diferentes sistemas del vehículo que han sufrido modificaciones e innovaciones debido a la implementación de la electrónica.

Figura 3. Sistemas del vehículo que han sido influenciados por la electrónica (Artero, 2016)

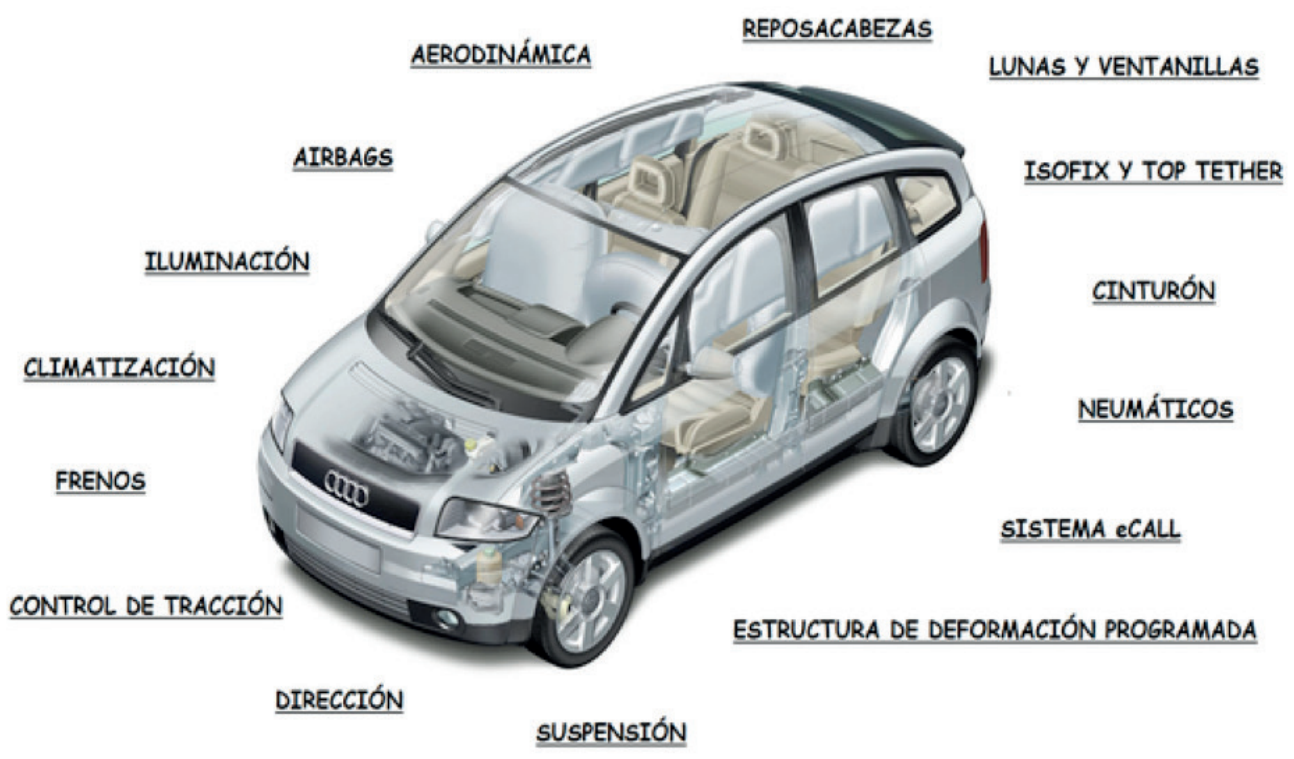


2.2.1. Electrónica y seguridad: elementos pasivos y activos; como ejemplos se citan los sensores de aceleración que actúan con los airbag y equipamiento electrónico para asistencia a la conducción, sistemas de posicionamiento GPS, detectores de distancia de otros vehículos y de ayuda a estacionar.

Además de los sistemas mecatrónicos de seguridad que actualmente se equipan, tales como sistemas de control de estabilidad, los automóviles comenzarán a incorporar sistemas de comunicación V2V, vehículo hacia vehículo, y V2I, vehículo con el entorno (Santa, Gómez y Sánchez, 2008).

2.2.2. Electrónica y sensores: las técnicas que se usan para recolectar las variables físicas de operación y comandos del conductor son variadas y las tecnologías involucradas son semiconductores de potencia, sensores micromecánicos y de aplicación específica, elementos de mecatrónica, y unidades electromecánicas, como ejemplo se nombran: sensores de posición, medidores de caudal, sensores de temperatura de efecto Hall, sensores de vibración piezoeléctricos, acelerómetros integrados, de presión MAP, y de control de movimiento.

2.2.3. Eficiencia energética: relacionado con sistemas como el control de encendido, tiempos y relación de la mezcla de la inyección electrónica. Por ejemplo, la inyección directa de la mezcla permite un aumento de la eficiencia en motores de una misma cilindrada debido a que controla tanto el momento de realizar la combustión, la razón combustible-aire, la distribución dentro del pistón y el encendido programado de la bujía.

2.2.4. Electrónica y la asistencia en la conducción: las acciones del conductor son asistidas con programas que supervisan y se adaptan al modo de conducir de cada uno. Es posible encontrar vehículos que ofrecen programas para diversas condiciones de conducción, ajuste de asientos y de clima, sistemas de sonido, etc., que se adaptan automáticamente a las preferencias del conductor.

2.2.5. Sistemas de transmisión: se han introducido las cajas manuales con accionamiento robotizado. Motores eléctricos y relés comandados en forma electrónica que realizan el cambio de engranaje, ya sea por acción directa del conductor o por acción de un computador de gestión de caja de cambio que brindan mejorar la eficiencia de las cajas manuales y las comodidades de las automáticas.

2.2.6. Sistemas de dirección: en cuanto a las mejoras relativas a la dirección se ha tenido una evolución desde las tradicionales direcciones mecánicas, direcciones hidráulicas o direcciones electrohidráulicas, hasta llegar a las actuales direcciones eléctricas, donde a pesar de toda la gestión electrónica, sigue existiendo una unión mecánica entre el volante y la cremallera de dirección, algo que llega a desaparecer en la dirección Steer-by-wire.

2.2.7. Sistemas de suspensión: en lo referente a la suspensión, durante los últimos años se han mejorado enormemente los sistemas de suspensión adaptativa y sistemas de control de balanceo.

2.2.8. ECU, o Electronic Control Unit: la introducción de las primeras ECU fueron la respuesta por parte de los fabricantes de automóviles americanos a las cada vez más exigentes regulaciones con respecto a la emisión de gases tóxicos de los automóviles. Debido al aumento de nuevas funciones y sistemas mecatrónicos en los nuevos vehículos, se habla de diversas ECU encargadas cada una de ellas de una función de manera específica. Todas estas unidades están centralizadas y comunicadas, por medio de un bus de datos o bus CAN, que es un protocolo de comunicación basado en un bus serie para el intercambio de información de las distintas unidades centrales. Al hacer componentes electrónicos más pequeños (microscópicos) ha 
permitido la integración de un mayor número de funciones en el interior de un mismo calculador o centralita (Lara, 2014). Un vehículo actualmente contiene alrededor de 25 a 35 microcontroladores, y los de lujo llegan a tener aproximadamente de 60 a 150 (Lucas, 2016).

2.2.9. La evolución relacionada con la transmisión de datos: se ha enmarcado en tres fases: la primera evolución, consiste en que los cables tienen como principal y exclusiva función conducir electricidad; la segunda, tiene relación cuando los cables conducen electricidad e información; este sistema se caracteriza por un cable/una señal; la tercera, se caracteriza porque el cable puede transmitir más de una señal (sistemas multiplexados) (Figueroa, 2015). Actualmente, hay cinco arquitecturas de red típicas utilizadas en la industria automotriz, estos incluyen: Controller Area Network (CAN)-CAN es la arquitectura de red más antigua. Desde que fue estandarizado por ISO 11898 en 1981, CAN puede haber proporcionado un bus serie de datos de baja velocidad, que va desde $1 \mathrm{~kb} / \mathrm{s}$ (kilobytes por segundo) a $1 \mathrm{Mb} / \mathrm{s}$ (millones de bytes por segundo) y goza de soporte de múltiples proveedores. Media Oriented Systems Transport (MOST): desarrollada en 2001, se utiliza para controlar y transmitir datos de infoentretenimiento. Puede funcionar a $25 \mathrm{Mb} / \mathrm{s}$ y $150 \mathrm{Mb} / \mathrm{s}$ sobre fibra óptica plástica costosa o a una velocidad más lenta de $50 \mathrm{Mb} / \mathrm{s}$ sobre el cable de cobre convencional. Low-Voltage Differential Signaling (LVDS): se introdujo en 1994 para ofrecer un ancho de banda más amplio de 1 a 4 Gb/s (1 Gb/s = 1,000 Mb/s) para cámaras y pantallas (Davó, 2012). FlexRay: desarrollado y lanzado por un consorcio de compañías automotrices en 2005 , este bus de datos en serie de $10 \mathrm{Mb} / \mathrm{s}$ se ha utilizado como control de bus para aplicaciones altamente dinámicas y control de chasis. Fue estandarizado por ISO 10681, goza de soporte de múltiples proveedores y es más interoperable que LVDS. Ethernet: Gigabit Ethernet: se ha utilizado desde finales de la década de 1990. Desde entonces, se ha convertido en una tecnología madura, con flexibilidad comprobada, escalabilidad, confiabilidad y otras ventajas, en comparación con las arquitecturas anteriores. Funciona a 100 Mb/s, y fue estandarizado por la ISO 13400-3 / Comunicación de diagnóstico por protocolo de Internet (DoIP).

2.2.10. Equipos de diagnóstico automotriz: se originan por la necesidad de reducir la contaminación de la atmósfera. Para que el conductor detecte un mal funcionamiento del OBD, se introdujo la obligación de tener una lámpara que indique fallos (MIL - Malfunction Indicator Lamp). Por ejemplo: en Europa, según la Directiva 98/69EG, los automóviles a gasolina del año 2000 en adelante; los vehículos a diésel de 2003 en adelante; y los camiones de 2005 en adelante, tienen que estar provistos de un OBD. La interfaz estándar del OBD-II no solamente es utilizada por el fabricante para sus funciones avanzadas de diagnóstico, sino también por aquellos que van más allá de lo que la ley exige. La siguiente etapa planeada es el OBD-III, en el que los propios automóviles se comunican con las autoridades si se produce un empeoramiento de las emisiones de gases nocivos mientras se está en marcha.

2.2.11. En lo relacionado a la conectividad se puede abordar lo relacionado a las conexiones internas. Sistemas de bus de datos para la interconexión de consola central con unidad de entretenimiento de asientos traseros, clúster de instrumentos digitales y otros módulos que por ejemplo puedan hacer streaming de multimedia en el vehículo y cuyo ancho de banda excede la capacidad del bus CAN y puede requerir como mínimo una red MOST de alta velocidad o AVB sobre Ethernet. Conexión a dispositivos portátiles Dispositivos con MTP (Media Transfer Protocol), iPods, reproductores MP4, dispositivos de almacenamiento masivo con conexión USB, auriculares Bluetooth, etc., hoy se dispone con móviles multimedia que pueden hacer streaming de contenidos o suministrar información de localización GPS, contactos de agenda o gestión de calendario. Conexión a la red o conexión a la nube con aplicaciones derivadas de la funciona- 
lidad de Pandora, Netflix, Hulu, Twitter o cualesquiera servicios basados en Internet mediante dispositivos de acceso a red (NAD) y una disponibilidad casi ubicua de redes inalámbricas que estará disponible con los sistemas 4G o LTE (García, 2015).

En 2013 y 2014 apareció CarPlay (Herrerías, 2016), la tecnología de Apple, y Android Auto, la solución que nos propone Google. De hecho, esta última empresa se lo ha tomado muy en serio desde el primer momento al involucrar en su proyecto a los fabricantes de coches a partir de la creación de un grupo de trabajo dedicado exclusivamente a facilitar la integración de Android en el coche: la Open Automotive Alliance.

Sistema E-call fue desarrollado a partir de 1 de octubre de 2015, el cual realiza una llamada a los servicios de emergencia automáticamente cuando se produce un accidente grave (debe dispararse al menos un airbag). Indica la posición exacta del vehículo mediante las coordenadas GPS, la dirección, la hora y el número de ocupantes.

Según un análisis realizado por Nishiguchi sobre la fabricación de vehículos en Japón y Estados Unidos enfocados en la parte mecánica y electrónica a través de la historia, se observa que hasta la década de 1960 un vehículo convencional estaba conformado básicamente por componentes mecánicos (Nishiguchi, 1994). La integración de componentes electrónicos se incrementa en los años setenta. Si se compara un vehículo típico de los años sesenta con los vehículos actuales, las diferencias cuantitativas son significativas, pero las transformaciones cualitativas son más profundas (Lara, 2014).

La naturaleza y estructura de los vehículos han desarrollado transformaciones en los componentes y en los sistemas mecatrónicos que se han instalado, como resultado de aquello casi el 90 \% de la innovación que se ha producido en la industria automotriz está relacionada con los componentes electrónicos, y de los cuales el 80 \% se relaciona con el software (Ordóñez, 2005).

2.2.12. Conducción Autónoma: es el desarrollo e incursión de nuevas tecnologías aplicadas en vehículos para lograr la autonomía del mismo, con el objetivo de conseguir que el vehículo obtenga la respuesta similar a la conducción de una persona en cualquier situación o medio. La presencia de esta tecnología en las calles supone una serie de ventajas y desventajas. La convivencia de los sistemas automáticos y los autos regulares, los desafíos legales y la seguridad de los pasajeros son solo algunas. Pero un aspecto que también están considerando las compañías automotrices son la comodidad de ser transportados al trabajo mientras repasamos la próxima reunión, leemos un buen libro o bien desayunamos, son algunas de las actividades que desarrollarán los pasajeros cuando estén siendo transportados por estos vehículos. Los principales elementos que transforman un coche "común" en un autónomo son los radares, sensores, cámaras e incluso el control electrónico de estabilidad. Para aclarar como el sistema trabaja en general, basta pensar en el funcionamiento del cuerpo humano: el coche también posee un cerebro que comanda las acciones en el vehículo. Los sensores hacen función de los ojos, oídos, tacto y, por último, los procesadores, que transforman informaciones en movimientos. En una persona, serían los brazos y las piernas. Al entender cómo funcionan y trabajan, usted también entiende un poco de lo que hace posible un coche caminar sin un conductor.

2.2.13. Car-to-X: Es un sistema que trata de que los automóviles sean capaces de comunicarse a través de redes inalámbricas de largo alcance. Entre las posibles aplicaciones del invento se encuentran, por ejemplo, ofrecer alertas de tráfico, la proximidad de vehículos de emergencia o información acerca de la meteorología, permitiendo que un automóvil avise directamente al resto de conductores conforme se vaya acercando a los mismos (Henriquez, 2018). Actualmente es común encontrar en ciudades sistemas de transporte inteligente, estos 
sistemas son generalmente de tipo web, y permiten al usuario conectado al Internet buscar una ruta entre dos puntos, con las respectivas relaciones de transporte público (Rojas, Bustos y Ordoñez, 2017).

\section{Conclusiones y recomendaciones}

Los avances relacionados con la electrónica han ocasionado un crecimiento acelerado de innovación del diseño y manufactura de los ECU, sensores, solenoides y arneses, así como a la administración del volumen de energía e información asociados a una red cada vez más extensa e interdependiente.

En la evolución de componentes electrónicos hay que tener en cuenta la innovación del resto de sistemas que conforman un automóvil, para lograr un funcionamiento armónico y eficiente del vehículo. Existen también sistemas que se destinan a mejorar la seguridad y las condiciones del tráfico, ofreciendo al conductor información sobre posibles obstáculos en el camino o dificultades en la vía.

En la medida que se integran más subsistemas mecatrónicos vinculados con distintas funciones de frenado, dirección, suspensión, etc., también se integran protocolos de comunicación de alta velocidad capaces de responder en tiempo real a los requerimientos especificados.

La evolución de la electrónica de consumo ha originado que los requisitos para las comunicaciones y las funciones de entretenimiento de los vehículos sean más exigentes, por lo que el desarrollo de control de seguridad y comunicaciones y electrónica de entretenimiento han tenido un rápido crecimiento.

Un vehículo autónomo puede contribuir a una conducción eficiente, pero a la vez crea un debate mundial en cuanto a la inteligencia artificial, por cuanto el humano se vuelve un ser dependiente de la tecnología.

Bajo la premisa de que los vehículos responden a una serie de condicionantes, la posible evolución de estos requisitos proporcionará las claves de las características de los automóviles del futuro. Se incorporarán todas aquellas funciones tecnológicas que aumenten la seguridad o la comodidad del usuario, siempre que no supongan incrementos de costes no deseables.

\section{Referencias}

Artero, C. (30 de Abril de 2016). aprendemergencias.es. Obtenido de https://www.aprendemergencias. es/seguridad-vial/sistemas-de-seguridad-en-el-veh\%C3\%ADculo/

Bonet, E. (2017). La revolución electrónica (Vol. 8). Ed. Universidad de Cantabria.

Cook, J., Sun, J., Buckland, J., Kolmanovsky, I., Peng, H., y Grizzle, J. (2006). PenAutomotive powertrain control-a survey. Asian Journal of Control, 8 (3), 237-260.

Davó, M. (2012). Desarrollo de aplicación de firmware para gestión de imágenes y de vídeo (Bachelor's thesis). Fernández, R. (2017). Estudio de mercado de la incorporación de sistemas de confort, asistencia y seguridad en los vehículos.

Fiarchild Semiconductor. (2012). Anual Report. San José: Cal: Fiarchild Semiconductor.

Figueroa, H. (2015). Estudio y análisis del sistema multiplexado del vehículo híbrido toyota prius.

García, I. (2015). Sistema colaborativo de notificaciones integrado en ordenador de a bordo.

Greenstein, S. (1997). What does industry convergence mean. Competing in the age of digital convergence. Henriquez, E. (4 de 05 de 2018). engadget. Obtenido de https://www.engadget.com/es/2011/10/24/carto-x-el-nuevo-sistema-de-comunicacion-entre-vehiculos-de-bm/ 
Herrerías, J. (2016). Conceptualització i disseny d'una aplicació d'oci basada en Android Auto.

Industria CCOO. (2018). Situación y perspectivas en el sector del automóvil, medidas ambientales, digitalización y automatización de la industria. Madrid: Area de Estrategicas Sectoriales. Obtenido de http://industria.ccoo.es/9ddeee3ef0745110d18ae92f9a4bc706000060.pdf

Lammers, D. (Julio de 2016). Nano Chip. Obtenido de http://www.appliedmaterials.com/nanochip/nanochip-fab-solutions/july-2016/cover-story-driving-innovation-cars-electronics-converge

Lara, R. (2014). De sistema mecánico a sistema tecnológico complejo: El caso de los automóviles. Contaduría y administración, 59 (2), 11-39.

Leen, G., y Heffernan, D. (2002). Expanding automotive electronic systems. Computer. 35 (1), 88-93.

Lucas, F. (2016). Software de simulación del comportamiento de sistemas de control de vehículos en función de parámetros electrónicos y medioambientales.

Nishiguchi, T. (1994). Strategic industrial sourcing: The Japanese advantage. Oxford University Press on Demand.

Ordóñez, S. (2005). Empresas y cadenas de valor en la industria electrónica en México: Electronics Firms and its Insertion in Value Chains. Economía UNAM, 2 (5), 90-111.

Parera, A. (2000). Sistemas de seguridad y confort en vehículos automóviles. Marcombo.

París, A. (2003). Aplicaciones electronicas del automovil 2. Vivat Academia, (25), 37-48.

Rojas, J., Bustos, J., y Ordoñez, D. (2017). Transporte público inteligente al alcance de sus manos. Enfoque UTE, 122-134.

Santa, J., Gómez, A., y Sánchez, M. (2008). Architecture and evaluation of a unified V2V and V2I communication system based on cellular networks. Computer Communications, 31 (12), 2850-2861.

Schöner, H. (2004). Automotive mechatronics. Control engineering practice, 12 (11), 1343-1351. 